\title{
GELFAND THEOREM IMPLIES STONE REPRESENTATION THEOREM OF BOOLEAN RINGS
}

\section{PARFENY P. SAWOROTNOW}

\author{
Department of Mathematics \\ The Catholic University of America \\ Washington, DC 20064 \\ United States of America
}

(Received June 27, 1994 and in revised form September 9, 1994)

\begin{abstract}
Stone Theorem about representing a Boolean algebra in terms of open-closed subsets of a topological space is a consequence of the Gelfand Theorem about representing a $B^{\star}$ algebra as the algebra of continuous functions on a compact Hausdorff space.
\end{abstract}

KEY WORDS AND PHRASES. Banach algebra, $B^{\star}$-algebra, Boolean algebra, Boolean ring, Stone Representation Theorem.

1991 AMS SUBJECT CLASSIFICATION CODES. Primary 06E15, 06E05, 46J25; Secondary 03G05, 46H15, 46J10

\section{INTRODUCTION.}

Gelfand Theorem in the title is the Representation Theorem for commutative normed rings with an involution, which can be stated as follows (in American terminology):

Representation Theorem of Gelfand. For each commutative (complex) $B^{\star}$-algebra $\mathcal{B}$, with identity, there exists a compact Hausdorff space $S$ such that $\mathcal{B}$ is isomorphic and isometric to the algebra $C(S)$ of all continuous complex valued functions on $S$ (see, for example, first corollary to the Theorem in $26 \mathrm{E}$ of Loomis [3] or Theorem A in section 73 of Simmons [7]).

The isomorphism preserves also the involution, i.e. $\hat{a^{\star}}=\overline{\hat{a}}$, where $\hat{a}$ is the member of $C(S)$ corresponding to $a$ (and $\hat{a^{\star}}$ corresponds to $a^{\star}$ ).

\{A $B^{\star}$-algebra (Sec. 72 of Simmons [7]) is a Banach algebra $\mathcal{B}$ with an involution $x \rightarrow x^{\star}$ such that $\left\|x^{\star} x\right\|=\|x\|^{2}$ for all $\left.x \in \mathcal{B}\right\}$.

2. MAIN RESULT.

In the sequel we shall establish validity of the following proposition:

THEOREM. Representation Theorem of Gelfand implies Stone's Representation Theorem for Boolean Rings (see Appendix Three of Simmons [7]): 
For each Boolean ring $\mathcal{A}$ there exists a totally disconnected compact Hausdorff space $S$ such that $\mathcal{A}$ is isomorphic to the Boolean ring $\mathcal{A}(\mathcal{S})$ of all open-closed subsets of $S$ (the operations of $\mathcal{A}(\mathcal{S})$ are the symmetric difference $A \triangle B=(A \cap \tilde{B}) \cup(\tilde{A} \cap B)$ and intersection $A \cap B, A \subset S, B \subset S)$.

PROOF. Assume validity of Gelfand Theorem. Let $\oplus$ and $\odot$ be operations of $\mathcal{A}$ and let 1 and 0 be, respectively, its multiplicative and additive identities. We shall say that a finite set $\left\{a_{1}, \ldots, a_{n}\right\}$ of members of $\mathcal{A}$ is a decomposition of identity if $a_{1} \oplus a_{2} \oplus \cdots \oplus a_{n}=1$ (below we shall write $\left.\sum_{i=1}^{n} \oplus a_{\imath}=1\right)$ and $a_{\imath} \odot a_{\jmath}=0$ if $i \neq j$.

For each decomposition $\left\{a_{1}, \ldots, a_{n}\right\}$ of identity and each set $\left\{\lambda_{1}, \ldots, \lambda_{n}\right\}$ of complex numbers consider a formal sum $f=\sum_{i=1}^{n} \lambda_{i} a_{i}$. Let $\mathcal{B}^{\prime}$ be the class of all such formal sums. Let us use the following notation: we shall write $[f]=\left\{a_{1}, \ldots, a_{n}\right\}$ and $\lambda_{2}=\lambda\left(a_{2}\right)$ if $f=\sum_{i=1}^{n} \lambda_{i} a_{2}$ is any member of $\mathcal{B}^{\prime}$.

Define relation " $\sim$ " on $\mathcal{B}^{\prime}$ as follows: $f \sim g$ if $\lambda(a)=\lambda(b)$ for any $a \in[f]$ and $b \in[g]$ such that $a \odot b \neq 0$. It is an equivalence relation. In fact, we only need to prove reflexivity.

Let $f \sim g$ and $g \sim h$, where $f=\sum \lambda_{\imath} a_{i}, g=\sum \mu, b_{j}$ and $h=\sum \nu_{k} c_{k}$. Assume that $a_{\mathfrak{i}} \odot c_{k} \neq 0$, then from the fact that $\sum \Phi b_{j}=1$ we conclude that there is some integer $j$ such that $a_{\imath} \odot c_{k} \odot b_{\jmath} \neq 0$. Then both $a_{\imath} \odot b_{\jmath} \neq 0$ and $b_{\jmath} \odot c_{k} \neq 0$ (note that $b_{\jmath} \odot b_{\jmath}=b_{\jmath}$ ), which implies $\lambda_{i}=\mu_{\jmath}=\nu_{k}$.

Now let us define addition, multiplication, multiplication with complex numbers and involution on $\mathcal{B}^{\prime}$ as follows: If $f=\sum \lambda_{2} a_{2}, g=\sum \mu, b$, and $\lambda$ is a scalar then

$$
\begin{aligned}
f+g & =\sum_{i, j}\left(\lambda_{\imath}+\mu_{3}\right) a_{\imath} \odot b_{j} \\
f g & =\sum_{i, j} \lambda_{\imath} \mu_{j} a_{\imath} \odot b_{J} \\
\lambda f & =\sum_{i} \lambda_{i} \lambda_{i}
\end{aligned}
$$

and

$$
f^{\star}=\sum_{\imath} \bar{\lambda}_{\imath} a_{\imath}
$$

It is easy to see that these operations are invariant under the relation " $\sim$ " i.e. " $f \sim g$ " implies " $f+h \sim g+h, f h \sim g h$ and $\lambda f \sim \lambda g$." $\{$ For example, if $f, g$ and $h$ are as above and $f \sim g$, then $f+h=\sum_{i, k}\left(\lambda_{i}+\nu_{k}\right) a_{i} \odot c_{k}$ and $g+h=\sum_{\jmath, k}\left(\mu_{\jmath}+\nu_{k}\right) b_{\jmath} \odot c_{k}$. If $\left(a_{\imath} \odot c_{k}\right) \odot\left(b_{\jmath} \odot c_{k^{\prime}}\right) \neq 0$, then $a_{i} \odot b_{j} \neq 0$ and $k=k^{\prime}$. This implies that $\lambda_{z}=\mu_{\jmath}$, and from this we conclude that $\left.f+h \sim g+h\right\}$.

Define the semi-norm \|\| on $\mathcal{B}^{\prime}$ by setting $\|f\|=\max \{|\lambda(a)|: a \in[f], a \neq 0\}$. Also it is easy to see that \|\| is invariant under the relation " " i.e. $f \sim g$ implies $\|f\|=\|g\|$.

Let $\tilde{\mathcal{B}}$ be the collection of all equivalence classes with respect to " $\sim$ ". Then $\tilde{\mathcal{B}}$ is a normed linear algebra with respect to the operations induced by operations on $\mathcal{B}^{\prime}$ (we shall use same notation), the additive identity $\overrightarrow{0}$ of $\tilde{\mathcal{B}}$ is the set of all members of $\mathcal{B}^{\prime}$ of the form $f=\sum_{2} \lambda_{2} a_{2}$, 
where $\left\{a_{1}, \ldots, a_{n}\right\}$ is some decomposition of identity and $\lambda_{2}=0$ for $i=1, \ldots, n$. Also $\|f\|=0$ if and only if $f=\overline{0}$. and it is not clifficult to show that $\|f g\| \leq\|f\| \cdot\|g\|$ for all $f, g \in \tilde{\mathcal{B}}$.

Let $\mathcal{B}$ be the completion of $\tilde{\mathcal{B}}$ with respect to \|\| . Then $\mathcal{B}$ is a commutative $B^{\star}$-algebra with identity.

Apply Representation Theorem of Gelfand to $\mathcal{B}$ : There exists a compact Hausdorff space $S$ such that $\mathcal{B}$ is $\star$-isomorphic and isometric to the algebra $C(S)$ of all continuous complex valued functions on $S$. For each $f \in \mathcal{B}$ let $\hat{f}(s)$ denote the corresponding image of $f$ under this isomorphism. Isometry between $\mathcal{B}$ and $C(S)$ means that $\|f\|=\sup _{s \in S}|\hat{f}(s)|$.

Now note that there is a natural imbedding of the Boolean ring $\mathcal{A}$ into $\mathcal{B}$ : for each $a \in \mathcal{A}$ let $f_{a}=1 \cdot a+0 \cdot a^{\prime}$, where $a^{\prime}=1 \varpi a$ is the complement of $a$ in $\mathcal{A}$. (Note that $\mathcal{A}$ has also a structure of a Boolean algebra (see Appendix Three of Simmons [7]).) Then $f_{a} f_{a}=f_{a}$, from which we conclude that $\hat{f}_{a}(s)$ assumes either 1 or 0 at any $s \in S$. Let $A=\left\{s \in S: f_{a}(s)=1\right\}$, then $\hat{f}_{a}(s)$ is the characteristic function of $A$ i.e. $\hat{f}_{a}=\varphi_{A}$, and it follows from continuity of $\hat{f}_{a}$ that $A$ is both open and closed in $S$. The correspondence $a \leftrightarrow A$ is $1-1$ and preserves both lattice and algebraic operations of $\mathcal{B}$. A simplest way to establish this is to show that this correspondence preserves multiplication and complementation. But both facts follow easily from the identities " $f_{a \odot b}=f_{a} f_{b}$ " and " $f_{a} f_{a^{\prime}}=0 "\left(a, b \in \mathcal{A}\right.$ and $\left.a^{\prime}=a \oplus 1\right)$.

It remains to show that $S$ is totally disconnected. Since $S$ is a Hausdorff space, we need only to show that for any open neighborhood $U$ of $s_{o} \in S$ there exists an open-closed set $O$ such that $s_{o} \in O \subset U$. Since every compact $T_{2}$ space is normal, there exists a continuous real valued function $x(s)$ (a member of $C^{\prime}(S)$ ) such that $x\left(s_{o}\right)=0, x(S \sim U)=1$ and $0 \leq x(s) \leq 1$ everywhere else (see Urysohn's Lemma in Sec. 3, Chap. 8 of Royden [6] or 3C in Loomis [3]). Let $x \in \mathcal{B}$ be such that $\hat{x}(s)=x(s)$ and let $f \in \mathcal{B}^{\prime}, f=\sum_{\imath=1}^{n} \lambda_{\imath} a_{\imath}$ be such that $\|f-x\|<\frac{1}{4}$. From " $\sum_{i=1}^{n} \oplus a_{\imath}=1$ and $a_{\imath} \odot a_{\jmath}=0$ if $i \neq j$ " we conclude that sets $A_{1}, A_{2}, \ldots, A_{n}$ (corresponding to $a_{1}, a_{2}, \ldots, a_{n}$ under above discussed correspondence $\left.a \leftrightarrow A\right)$ are disjoint and $\cup_{\imath} A_{\imath}=S$. Hence there exists exactly one index $j \in(1,2, \ldots, n)$ such that $s_{o} \in A_{\jmath}$. The set $A$, is both open and closed and $s \in A_{\jmath}$ implies $\hat{f}(s)=\lambda_{\jmath}=\hat{f}\left(s_{o}\right)$, from which we conclude that $A_{\jmath} \subset U:$ if $s \in A_{\jmath}$, then $|x(s)| \leq|x(s)-\hat{f}(s)|+\left|\hat{f}\left(s_{o}\right)-x\left(s_{o}\right)\right|+\left|x\left(s_{o}\right)\right|<1$, and this implies that $s \in U$.

To see that each open-closed subset $A$ of $S$ corresponds to some $a \in \mathcal{A}$ we use compactness of $S$, which implies compactness of $A$. As above, for each $s \in A$ we select an open-closed set $O$ s such that $s \in O_{s} \subset A$. Compactness of $A$ implies that there is a finite set $\left\{A_{1}, \ldots A_{m}\right\}$ of open-closed subsets of $S$, each corresponding to some $a_{\imath} \in \mathcal{A}(i=1 \ldots m)$, such that $A=\bigcup_{\imath=1}^{m} A_{\imath}$. This implies that $A$ corresponds to some $a \in \mathcal{A}\left(a=a_{1} \cup a_{2} \ldots \cup a_{m}\right)$.

\section{REFERENCES}

1. GELFAND, I.M., RAIIOV, D.A. and ŠILOV, G.E. Commutative Normed Rings, Moscow 1960. 
2. IOOPPELBERT, S. Handlbook of Boolean Algebras, Vol. 1, North-Holland, 1989.

3. LOOMIS, L.H., An Introduction to Abstract Harmonic Analysis, Van Nostrand, 1953.

4. MACliEY, G.W., Commutative Banach Algebras, lecture notes, Harvard University, 1952.

5. NAIMARK, M. A., Normed Rings, Publisher "Nauka", Moscow, 1968.

6. ROYDEN, H.L., Real Analysis, MacMillen Publishing Co., New York, 1988.

7. SIMMONS, George F., Introduction to Topologv and Modern Analysis, McGraw-Hill, 1963. 


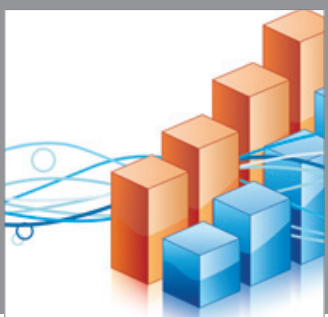

Advances in

Operations Research

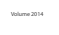

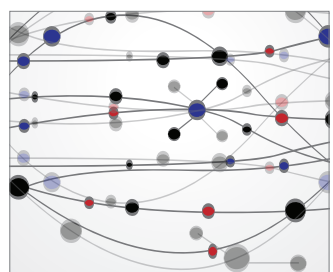

\section{The Scientific} World Journal
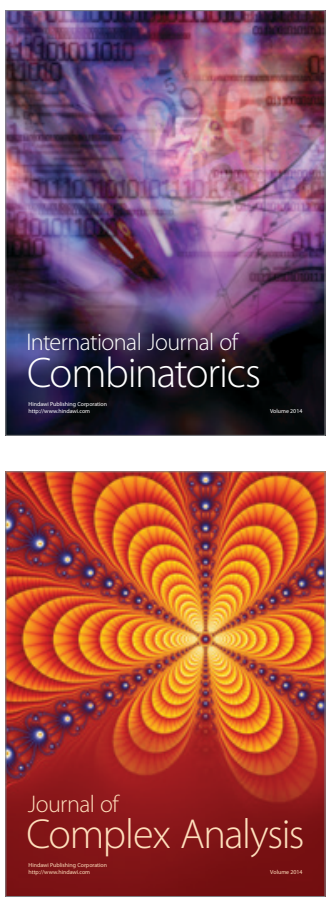

International Journal of

Mathematics and

Mathematical

Sciences
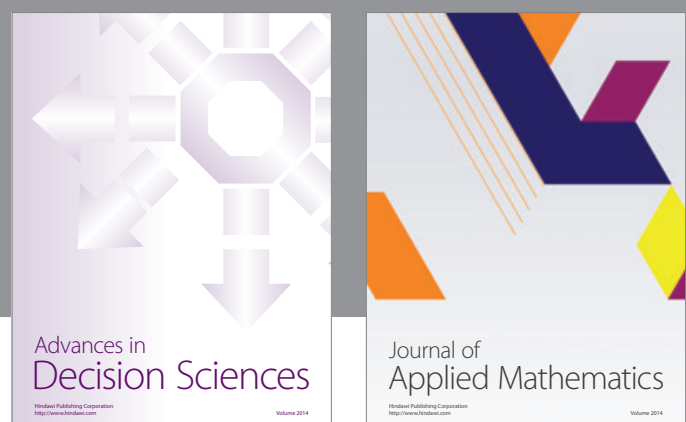

Journal of

Applied Mathematics
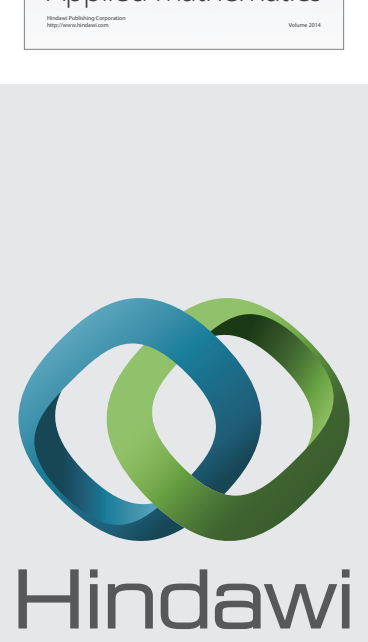

Submit your manuscripts at http://www.hindawi.com
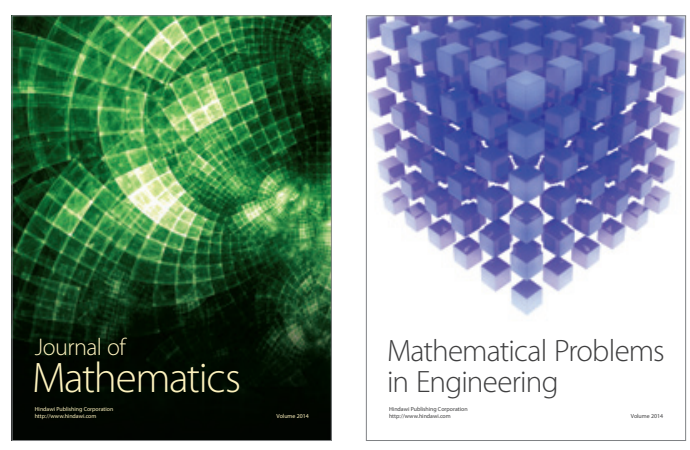

Mathematical Problems in Engineering
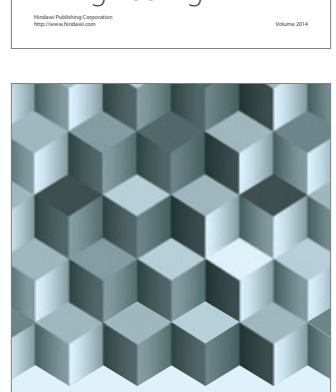

Journal of

Function Spaces
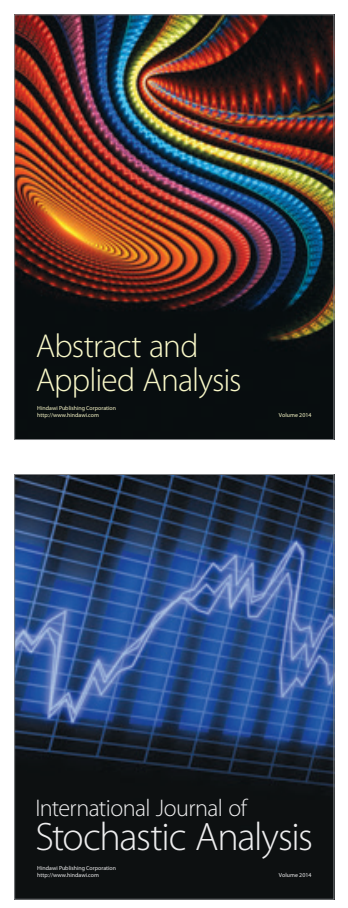

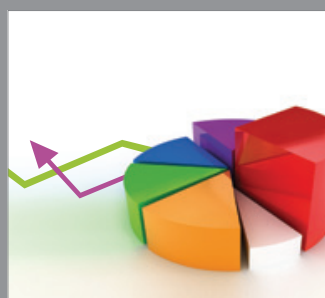

ournal of

Probability and Statistics

Promensencen
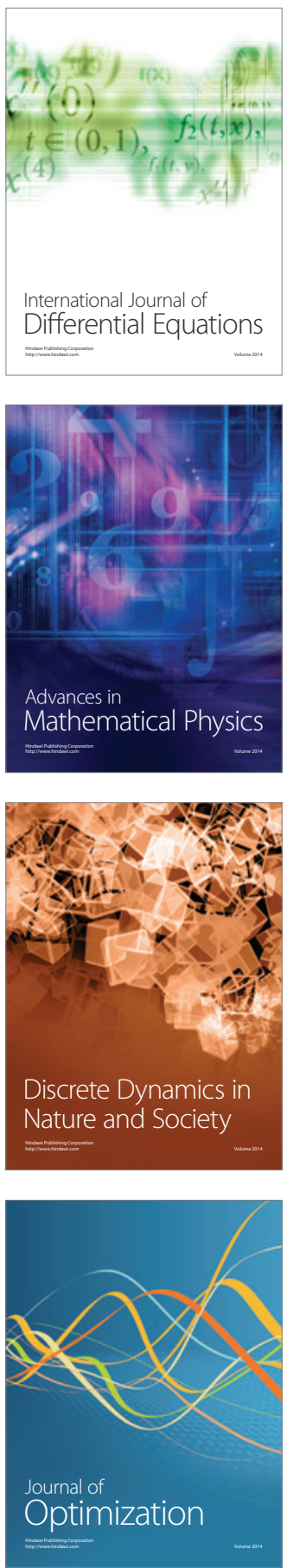